\title{
Antigen-Drug Conjugates as a Novel Therapeutic Class for the Treatment of Antigen-Specific Autoimmune Disorders
}

\author{
Chad J. Pickens ${ }^{1, \ddagger}$, Matthew A. Christopher ${ }^{1, \ddagger}$, Martin A. Leon ${ }^{2}$, Melissa M. Pressnall ${ }^{1}$, \\ Stephanie N. Johnson ${ }^{1}$, Sharadvi Thati ${ }^{1}$, Bradley P. Sullivan ${ }^{1}$, Cory Berkland ${ }^{1,}{ }^{*}$ \\ ${ }^{1}$ Department of Pharmaceutical Chemistry, University of Kansas, 2095 Constant Avenue, \\ Lawrence, Kansas 66047, USA \\ ${ }^{2}$ Department of Chemistry, University of Kansas, 1567 Irving Hill Rd, Lawrence, KS 66045, USA
}

\begin{abstract}
Multiple sclerosis represents the world's most common cause of neurological disability in young people and is attributed to a loss of immune tolerance toward proteins of the myelin sheath. Typical treatment options for MS patients involve immunomodulatory drugs, which act nonspecifically, resulting in global immunosuppression. The study discussed herein aims to demonstrate the efficacy of antigen-specific immunotherapies involving conjugation of disease causing auto-antigen, PLP139-151, and a potent immunosuppressant, dexamethasone. Antigendrug conjugates (AgDCs) were formed using copper-catalyzed azide-alkyne cycloaddition chemistry with the inclusion of a hydrolyzable linker to maintain activity of released dexamethasone. Subcutaneous administration of this antigen-drug conjugate to SJL mice induced with experimental autoimmune encephalomyelitis protected the mice from symptom onset throughout the 25-day study, demonstrating enhanced efficacy in comparison to dexamethasone treatment. These results highlight the benefits of co-delivery of auto-antigens with immunosuppressant drugs as $\mathrm{AgDCs}$ for the treatment of autoimmune diseases.
\end{abstract}

\section{Graphical Abstract}

\footnotetext{
*To whom correspondence should be addressed: University of Kansas, 2030 Becker Drive, Lawrence, KS 66047. Phone: (785) 864-1455, Fax: (785) 864-1454, berkland@ku.edu.

Fuuthors contributed equally to this work

Supporting Information

Figure S1. HSQC NMR data for DEX-N3. Figure S2. Levels of IFN- $\gamma$ at peak of disease. Figure S3. Levels of IL-6 at peak of disease. Figure S4. Levels of IL-15 at peak of disease. Figure S5. Levels of IL-17A at peak of disease. Figure S6. Levels of IL-21 at peak of disease. Figure S7. Levels of IL-23 at peak of disease. Figure S8. Levels of TNF-a at peak of disease.
} 


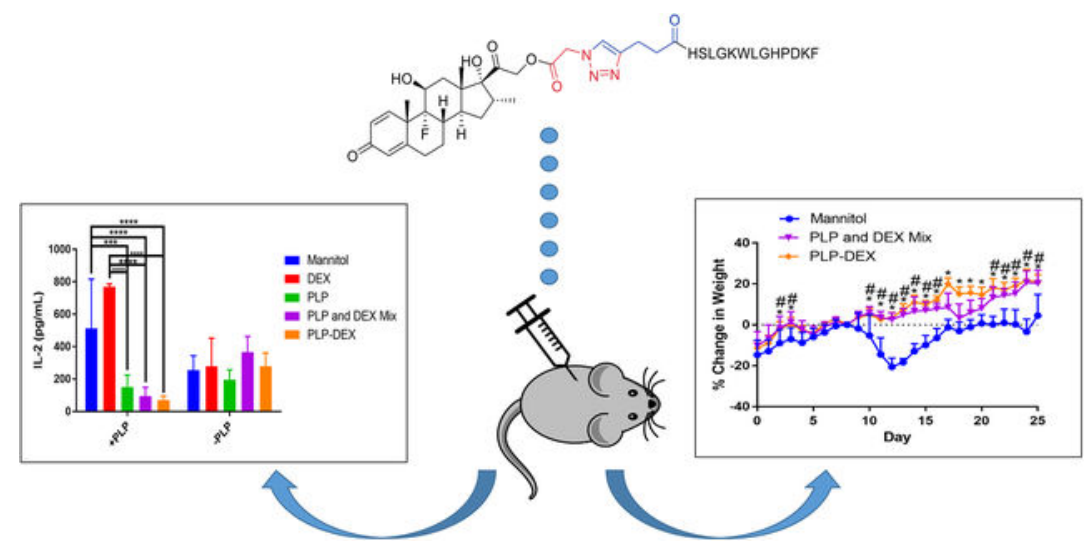

\section{Keywords}

Autoimmunity; antigen-specific immunotherapy; copper-catalyzed azide-alkyne cycloaddition; experimental autoimmune encephalomyelitis; antigen-drug conjugate

\section{Introduction}

The adaptive immune response is dependent on recognition of target antigens, and autoimmune diseases occur when the body fails to maintain tolerance toward self-antigens. ${ }^{1}$ These self-antigens can be present in a number of host tissues in various organs, leading to many types of autoimmune diseases including multiple sclerosis (MS). ${ }^{2} \mathrm{MS}$ is the most common cause of neurological disability in young adults, affecting approximately 2.5 million patients worldwide ${ }^{3}$, and symptoms of the disease are highly variable ${ }^{4}$ While the mechanisms of disease induction have not been fully elucidated, a prevailing hypothesis is that loss of immune tolerance towards proteins composing the myelin sheath, such as proteolipid protein (PLP) and myelin basic protein (MBP), triggers recruitment of offending myelin-specific $\mathrm{CD} 4^{+} \mathrm{T}$ cells, resulting in demyelination within the central nervous system. ${ }^{5}$ Current therapies include monoclonal antibodies and corticosteroids, however, the treatment landscape for MS is expanding as our understanding of disease pathogenesis continues to evolve. ${ }^{6}$ Corticosteroids have been a cornerstone for MS therapy, but the lack of specificity results in a wide range of side effects. Attempts have been made to develop antigen-specific immune therapies (ASITs) to reduce side effects and selectively re-tolerize the immune system to myelin sheath proteins. Despite being relatively safe, ASITs have yet to reduce the severity of MS. ${ }^{7-9}$ Here, we present antigen-drug conjugates (AgDCs) as a combination of these approaches with the potential to boost the efficacy of ASIT while mitigating the detrimental side effects of current immunosuppressant therapies.

AgDCs merge two general approaches to combat autoimmunity: immunomodulatory agents and ASIT. ASIT provides the specificity necessary to reverse an immune response toward a particular antigen. ASIT has been effective for inducing tolerance towards allergens, but has yet to emerge as an effective autoimmune therapy. ${ }^{7-9}$ Immunomodulatory agents can effectively treat autoimmune diseases, but suffer from systemic exposure and subsequent global immunosuppression, which can be problematic in immunocompromised patient 
populations by increasing vulnerability to opportunistic infections. By chemically conjugating the antigen and immunomodulator, the antigen may target the immunomodulator to diseased cell populations, potentially limiting off-target side effects. The concept of AgDCs is similar to antibody-drug conjugates (ADCs), however AgDCs likely achieve high affinity specificity by targeting endogenous autoantibodies or cognate B cell receptors, thus essentially flipping the mechanism of ADCs.

The design of AgDCs requires selection of an appropriate antigen or epitope, an immune modulator, and a linking scheme. With this in mind, a murine experimental autoimmune encephalomyelitis (EAE) model exhibiting clinical and histopathological similarities to relapsing-remitting multiple sclerosis (RRMS) in humans ${ }^{2}$ was selected to probe the proposed AgDC concept. The model is induced by administering an adjuvanted 'vaccine' containing the antigenic epitope PLP139-151. This particular EAE model is CD4+ $\mathrm{T}$ cellmediated disease with $\mathrm{B}$ cell involvement, leading to primary demyelination of the axonal tracks in the CNS and subsequent progressive paralysis of the hind-limbs. ${ }^{10-11}$ Most importantly, this particular EAE model provided a simplified system for testing the efficacy of AgDCs wherein the disease may be induced with a specific peptide, PLP139-151, and subsequently treated with an AgDC utilizing the same PLP139-151 epitope.

Dexamethasone (DEX) was selected as the immune modulator for testing the AgDC concept. Our lab previously screened multiple immune modulators in combination with PLP139-151 by using splenocytes derived from EAE mice. ${ }^{12}$ DEX emerged as one of the most potent suppressors of proinflammatory cytokines when rechallenging EAE splenocytes with PLP139-151 and also showed evidence of inducing markers of immune tolerance (e.g. upregulation of IL-10). DEX possesses the necessary potency required (pMol-nMol range $)^{13-14}$, since the dose typically used for antigen-specific immunotherapy is on the order of milligrams of antigen per injection. ${ }^{15}$ Finally, we rationalized DEX must be released in order to escape the typical binding and internalization pathway associated with antigen recognition and processing. Thus, we designed an ester linker capable of releasing DEX from PLP139-151 via hydrolysis with accelerated release under acidic conditions.

In this article, we outline the synthetic strategy, characterization, and biological screening of an AgDC containing PLP139-151 and DEX. These two components were conjugated utilizing copper-catalyzed azide-alkyne cycloaddition (CuAAC) and this linkage was characterized by HPLC and mass spectrometry. Additionally, AgDC linker stability was monitored over a short time frame to confirm the desired release of DEX. Finally, the efficacy of this AgDC was demonstrated first in vitro through the use of EAE splenocytes induced against PLP139-151 and subsequently in vivo through subcutaneous administration to EAE mice.

\section{Materials and Methods}

DEX, tris(3-hydroxypropyltriazolylmethyl)amine (THPTA), and sodium ascorbate (NaAsc) were purchased from Sigma-Aldrich (St. Louis, MO). Copper(II) sulfate pentahydrate $\left(\mathrm{CuSO}_{4} \cdot 5 \mathrm{H}_{2} \mathrm{O}\right)$ was purchased from Acros Organics (Geel, Belgium). 2,5dioxopyrrolidin-1-yl 2-azidoacetate, $\mathrm{DBCO}-\mathrm{PEG}_{4}$-Maleimide, DBCO-NH${ }_{2}$, and MMAE- 
DBCO were purchased from Click Chemistry Tools, LLC (Scottsdale, AZ). Doxorubicin hydrochloride was purchased from LC Laboratories (Woburn, MA). Mertansine (DM1) was purchased from Carbosynth Limited (Berkshire, UK). All other chemicals and reagents were analytical grade and were used as received without further purification.

The peptides PLP139-151-Alk and PLP139-151-N $\mathrm{N}_{3}$ have been synthesized in our laboratory via solid phase peptide synthesis on a Wang resin, but larger quantities of each peptide were obtained from Biomatik Corporation (Wilmington, DE). In each case, the linker 3-(2-(2-(2azidoethoxy)ethoxy)ethoxy)propanoic acid was purchased from PurePEG, LLC (San Diego, CA) and 4-pentynoic acid was purchased from Sigma-Aldrich (St. Louis, MO).

\section{Synthesis of azide-functionalized DEX $\left(\right.$ DEX- $\left.\mathrm{N}_{3}\right)$}

DEX was added to a flame dried $250 \mathrm{~mL}$ round bottom flask with a stir bar and septa. Anhydrous $\mathrm{MeCN}$ was added under nitrogen, then DIPEA via glass syringe. The flask was stirred for $10 \mathrm{~min}$ before azidoacetic acid NHS ester was added as a powder. The reaction mixture was stirred overnight at room temperature before being analyzed by HPLC. Additional equimolar aliquots of azidoacetic acid NHS ester were added, followed by stirring for 2 hours at room temperature and analyzing by HPLC, until no additional benefit was observed. The crude reaction mixture was evaporated under reduced pressure, then dissolved in 4:6 MeCN: $\mathrm{H}_{2} \mathrm{O}$ and purified by prep HPLC. The resulting column fractions were evaporated under reduced pressure to yield the final product as a white powder. ${ }^{1} \mathrm{H}$ NMR (500 MHz, DMSO-d 6 ) $\delta 7.30(\mathrm{~d}, \mathrm{~J}=10.2 \mathrm{~Hz}, 1 \mathrm{H}), 6.23(\mathrm{dd}, \mathrm{J}=10.1,1.9 \mathrm{~Hz}, 1 \mathrm{H})$, $6.01(\mathrm{t}, \mathrm{J}=1.7 \mathrm{~Hz}, 1 \mathrm{H}), 5.45(\mathrm{dd}, \mathrm{J}=5.0,1.4 \mathrm{~Hz}, 1 \mathrm{H}), 5.23(\mathrm{~s}, 1 \mathrm{H}), 5.17(\mathrm{~d}, \mathrm{~J}=17.5 \mathrm{~Hz}$, $1 \mathrm{H}), 4.90(\mathrm{~d}, \mathrm{~J}=17.6 \mathrm{~Hz}, 1 \mathrm{H}), 4.32-4.19(\mathrm{~m}, 2 \mathrm{H}), 4.19-4.11(\mathrm{~m}, 1 \mathrm{H}), 2.88(\mathrm{dqd}, \mathrm{J}=11.5$, 7.2, $4.1 \mathrm{~Hz}, 1 \mathrm{H}), 2.62(\mathrm{tdd}, \mathrm{J}=13.6,6.0,1.7 \mathrm{~Hz}, 1 \mathrm{H}), 2.44-2.32(\mathrm{~m}, 1 \mathrm{H}), 2.35-2.28(\mathrm{~m}$, $1 \mathrm{H}), 2.22-2.05(\mathrm{~m}, 3 \mathrm{H}), 1.77(\mathrm{dt}, \mathrm{J}=11.2,5.2 \mathrm{~Hz}, 1 \mathrm{H}), 1.70-1.58(\mathrm{~m}, 1 \mathrm{H}), 1.56(\mathrm{dd}, \mathrm{J}=$ $13.8,2.0 \mathrm{~Hz}, 1 \mathrm{H}), 1.49$ (s, 3H), 1.35 (qd, J = 12.9, $5.0 \mathrm{~Hz}, 1 \mathrm{H}$ ), 1.08 (ddd, J = 12.1, 8.2, 4.1 $\mathrm{Hz}, 1 \mathrm{H}), 0.89(\mathrm{~s}, 3 \mathrm{H}), 0.80(\mathrm{~d}, \mathrm{~J}=7.2 \mathrm{~Hz}, 3 \mathrm{H}) .{ }^{13} \mathrm{C}$ NMR $(126 \mathrm{MHz}$, DMSO) $\delta 204.36$, 185.30, 168.28, 167.10, 152.77, 129.03, 124.12, 102.00, 100.61, 90.52, 70.63, 70.34, 69.00, $49.34,48.09,48.05,47.87,43.33,35.69,35.53,33.67,33.51,31.92,30.28,27.32,23.03$, $22.98,16.31,15.15,1.19$. Expected $[\mathrm{M}+\mathrm{H}]^{+}=476.2191 \mathrm{Da}$, Observed $[\mathrm{M}+\mathrm{H}]^{+}=476.2067$ Da.

\section{Synthesis of PLP139-151-DEX}

To a solution of PLP139-151-Alk $(93.6 \mu \mathrm{mol})$ in $120 \mathrm{~mL}$ deionized $\mathrm{H}_{2} \mathrm{O}$ was added DEX$\mathrm{N}_{3}\left(189.4 \mu \mathrm{mol}, 2.02\right.$ eq.) in $12 \mathrm{~mL} \mathrm{EtOH} .5 .7 \mathrm{~mL}$ of a premixed solution of $\mathrm{CuSO}_{4} \cdot 5 \mathrm{H}_{2} \mathrm{O}$ $(38.1 \mu \mathrm{mol})$ and THPTA $(189.8 \mu \mathrm{mol})$ in deionized $\mathrm{H}_{2} \mathrm{O}$ was added to the reaction mixture, followed by $7.2 \mathrm{~mL}$ of NaAsc $(726.9 \mu \mathrm{mol})$ in deionized $\mathrm{H}_{2} \mathrm{O}$. The reaction was allowed to stir at room temperature for 1 hour before an aliquot was removed for analytical HPLC to monitor reaction progress. After 3 hours, the reaction mixture was concentrated under reduced pressure, and purified by preparative HPLC on a Waters XBridge BEH $\mathrm{C}_{18}, 5 \mu \mathrm{m}$, $130 \AA, 19 \times 250 \mathrm{~mm}$ column using a gradient of $\mathrm{MeCN}$ in $\mathrm{H}_{2} \mathrm{O}$ (constant $0.05 \%$ TFA). The isolated fractions were evaporated under reduced pressure to remove residual $\mathrm{MeCN}$, then frozen at $-20^{\circ} \mathrm{C}$ and lyophilized to yield a white powder. Expected $[\mathrm{M}+2 \mathrm{H}]^{2+}=1039.5224$ 
$\mathrm{Da},[\mathrm{M}+3 \mathrm{H}]^{3+}=693.3507 \mathrm{Da}$; Observed $[\mathrm{M}+2 \mathrm{H}]^{2+}=1039.5145 \mathrm{Da},[\mathrm{M}+3 \mathrm{H}]^{3+}=693.3478$

Da.

\section{Analytical characterization}

All HPLC chromatographic analysis was conducted on a Waters Alliance HPLC system equipped with either a diode array detector or dual wavelength UV/Vis detector. For RPHPLC, general chromatographic conditions employed a linear elution gradient from 5-95\% acetonitrile in water (constant $0.05 \%$ trifluoroacetic acid) over $50 \mathrm{~min}$, on a Waters XBridge BEH $\mathrm{C}_{18}, 3.5 \mu \mathrm{m}, 130 \AA$ A stationary phase $(4.6 \times 150 \mathrm{~mm})$, with a $1.0 \mathrm{~mL} / \mathrm{min}$ flow rate and a $35^{\circ} \mathrm{C}$ column temperature. For semi-preparative HPLC, a linear elution gradient of acetonitrile in water (constant $0.05 \%$ trifluoroacetic acid) over $20 \mathrm{~min}$, on a Waters XBridge BEH C $18,5 \mu \mathrm{m}, 130 \AA$ stationary phase $(19 \times 250 \mathrm{~mm})$, with a $14.0 \mathrm{~mL} / \mathrm{min}$ flow rate. Gradients were optimized for each run using the identical stationary phase in a $4.6 \times 250$ mm configuration.

LC/MS sample analysis was completed on a Waters Xevo G2, employing linear elution gradients of $15-100 \%$ acetonitrile in water (constant $0.1 \%$ formic acid) over $45 \mathrm{~min}$, on a Waters XBridge $\mathrm{BEH} \mathrm{C}_{18}, 1.7 \mu \mathrm{m}, 130 \AA$ 践 stationary phase $(0.075 \times 250 \mathrm{~mm})$, with a 0.5 $\mu \mathrm{L} / \mathrm{min}$ flow rate and $50^{\circ} \mathrm{C}$ column temperature. Electrospray ionization, operating in the positive mode (ESI+), was used as the ionization source with a QTOF mass analyzer used for detection.

NMR spectra were collected on a Bruker Avance AVIII $500 \mathrm{MHz}$ spectrometer equipped with a dual carbon/proton cryoprobe, and all samples were dissolved in $650 \mu \mathrm{L}$ of $\mathrm{D}_{2} \mathrm{O}$ or DMSO- $d_{6}$. Data processing was completed using MestReNova 11.0 (Santiago de Compostela, Spain).

\section{Drug release and stability studies}

Release and stability studies were conducted via HPLC with UV detection by dissolving the compound of interest in various buffers (typically $\mathrm{pH} 7.0$ phosphate, $\mathrm{pH} 5.5$ acetate, deionized $\mathrm{H}_{2} \mathrm{O}$, etc.) and evaluating the release at temperatures and times relevant to the particular study. Peak integration is used for quantification, accounting for initial purity of the PLP-DEX at $\mathrm{T}=0$.

\section{Induction of EAE}

In vitro and in vivo studies were performed through the use of 4-6 week old SJL/J $(\mathrm{H}-2)$ female mice purchased from Envigo Laboratories (Indianapolis, IN). All experiments were approved through the University's Institutional Animal Care and Use Committee with animal housing in pathogen-free conditions. An emulsion containing $200 \mu \mathrm{g}$ PLP139-151 in Complete Freund's Adjuvant (CFA) was prepared by combining IFA and heat-killed $M$. Tuberculosis strain H37RA at a final concentration of $4 \mathrm{mg} / \mathrm{mL}$ with subsequent emulsification between CFA and PBS containing $200 \mu \mathrm{g}$ PLP139-151. This PLP in CFA emulsion was administered to mice on day 0 through four subcutaneous injections of $50 \mu \mathrm{L}$ each located above each shoulder and hind flank for a total of $0.2 \mathrm{~mL}$. Additionally, intraperitoneal injections of pertussis toxin (100 ng in $100 \mu \mathrm{L}$ PBS) were administered on 
day 0 and day 2. Disease severity was recorded using the following clinical scoring system: 0 , no clinical disease symptoms; 1 , weakness or limpness of the tail; 2 , weakness or partial paralysis of one or two hind limbs (paraparesis); 3 , full paralysis of both hind limbs (paraplegia); 4, paraplegia plus weakness or paralysis of forelimbs; 5, moribund (euthanasia necessary). Mouse body weight was also recorded daily throughout the duration of the studies.

\section{In Vivo Efficacy in EAE Mice}

In vivo studies were performed with 9 mice per treatment group. Five mice were euthanized on day 14 (peak-of-disease) while the remaining mice were euthanized on day 25.

Treatments were administered to mice through subcutaneous injection of $100 \mu \mathrm{L}$ at the base of the neck on the back of the mouse on days 4, 7, and 10 following EAE induction. All treatments were dosed at a $200 \mathrm{nmol}$ DEX basis in $40 \mathrm{mg} / \mathrm{mL}$ mannitol as vehicle. Mice were weighed daily from day 0 and scored daily from day 7 until the end of the study at day 25.

\section{Splenocyte Isolation}

Spleens were harvested from EAE and healthy control mice on peak-of-disease or day 25 for in vitro and in vivo studies, respectively. Spleens were placed in $5 \mathrm{~mL}$ of RPMI 1640 containing L-glutamine and 1\% Penicillin-Streptomycin and placed on ice for transportation. The spleens were pressed through sterile wire mesh with the use of a rubber $1 \mathrm{~mL}$ syringe plunger and the cellular extract was collected and centrifuged at 1100xg for 5 minutes. In order to lyse red blood cells the cell pellet was resuspended in $5 \mathrm{~mL}$ of Gey's lysis solution for 5 minutes on ice. Quenching of the lysis solution was performed by adding $10 \mathrm{~mL}$ of RPMI 1640 supplemented with L-glutamine, 1\% Penicillin-Streptomycin, and 10\% fetal bovine serum (FBS) (complete RPMI, cRPMI). Cells were centrifuged at 1100xg for 5 minutes and resuspended in cRPMI prior to counting in $0.04 \%$ trypan blue. Cultures with in vitro treatments or PLP rechallenge were kept at $37^{\circ} \mathrm{C}$ and $5 \% \mathrm{CO}_{2}$.

\section{Cell Metabolic Assay}

Cellular metabolism was determined through the use of resazurin. Splenocytes were incubated with $75 \mu \mathrm{mol} / \mathrm{L}$ resazurin for 3 hours. Change in fluorescence (ex 560/em 590) provides a measurement of metabolic reductive capacity. Results for in vitro treated samples were normalized against untreated controls and background fluorescence was subtracted from all samples through fluorescence measurements on cRPMI containing resazurin. These readings were performed using a Spectramax M5 (Molecular Devices) plate reader.

\section{Cytokine Response}

Following the day 14 and day 25 splenocyte harvests from in vivo studies, in vitro splenocyte supernatants were collected after 96 hours incubation with PLP. Incubation was performed at $1 \times 10^{6}$ cells/well in 96 well plates in the presence or absence of $25 \mu \mathrm{M}$ PLP. The secretion of GM-CSF, IFN- $\gamma$, TNF- $a$ IL-2, IL-6, IL-10, IL-15, IL-17, IL-21, and IL-23 was measured using a U-Plex kit following manufacturer instructions (Meso Scale Discovery). 


\section{Statistical Analysis}

Statistical analysis of data was performed using two-way analysis of variance (ANOVA) and Tukey multiple comparison tests. $\mathrm{IC}_{50}$ studies were analyzed using a 4PL-sigmoidal function. Criteria for significance are as follows: $* \mathrm{p}<0.05$, ** $\mathrm{p}<0.01, * * * \mathrm{p}<0.001$, **** $\mathrm{p}<0.0001$. For Figure 7 and Figure 9 significant differences between the PLP and DEX mixture and mannitol vehicle control are denoted by \# $\mathrm{p}<0.05$. All statistical analysis was performed using GraphPad Software (GraphPad Software Inc.).

\section{Results}

\section{Analytical characterization of chemical entities}

The constituent and conjugate molecules can be characterized by traditional chemical methods, including NMR, HPLC, LC/MS, and other spectroscopic means to confirm the structure of the entity. HPLC analysis of PLP-Dex with UV/Vis detection provides typical purity values in excess of $93 \%$ following preparative HPLC purification (Figure 2). A similar analysis by LC-MS corroborates these results, showing a mass shift in the final conjugate indicative of attachment of DEX to PLP (Figure 3). From an NMR perspective, an added benefit to installing the alkyne linker on PLP is the presence of a distinct resonance in ${ }^{1} \mathrm{H} /{ }^{13} \mathrm{C}$ heteronuclear single quantum coherence (HSQC) experiment, corresponding to the terminal alkyne correlation $\left(\delta\left({ }^{1} \mathrm{H}\right) \approx 2.3 \mathrm{ppm}, \delta\left({ }^{13} \mathrm{C}\right) \approx 70 \mathrm{ppm}\right)$ which is present in a unique chemical environment and well separated from other signals (Figures 4 and S1). After conjugation, this resonance undergoes a significant downfield shift $\left(\delta\left({ }^{1} \mathrm{H}\right) \approx 7.8 \mathrm{ppm}\right)$ as it is incorporated as part of the conjugated triazole ring system (Figure 4). One additional advantage of conjugating hydrophobic drug molecules to hydrophilic peptides is the impact on aqueous solubility. The PLP139-151 peptide is soluble in excess of $60 \mathrm{mg} / \mathrm{mL}$, providing a significant enhancement in drug solubility in the resulting amphiphilic conjugates.

\section{Conjugate stability and drug release kinetics}

PLP-DEX contains an acid-labile ester linkage capable of releasing the unadulterated parent drug in acidic microenvironments present inside cells. A study of the release kinetics (Figure 5) indicated DEX is released over greater than 100 hours in unbuffered solutions and is released completely over approximately 50 hours when in acidic conditions (pH 5.5). Interestingly, complete hydrolysis of the linker occurs in a few hours when in phosphate buffered solutions, indicating that phosphate anions catalyze the release of DEX from PLP, likely through nucleophilic attack on the ester bond. Due to this finding, in vivo administration of PLP-DEX was carried out in $40 \mathrm{mg} / \mathrm{mL}$ mannitol to prevent hydrolysis prior to injection.

\section{$\mathrm{IC}_{50}$ determination of conjugates and payload}

In order to determine the impact of the CuAAC chemical linkage on the activity of esterified DEX, EAE splenocytes harvested at peak of disease (Day 12) were isolated and treated with DEX or PLP-DEX over a range of concentrations. After 120 hours, a resazurin assay was performed to assess cellular metabolism and these data were normalized to untreated EAE splenocytes (Figure 6). These data provide calculated IC50s for DEX and PLP-DEX of 6.84 
$+/-4.18 \mathrm{nM}$ and $7.55+/-2.80 \mathrm{nM}$, respectively. This result indicates that DEX maintains activity after release from the $\mathrm{AgDC}$ and demonstrates the rapid release of DEX from the acid-labile ester linkage in an in vitro setting, as evidenced by the similar IC50 values obtained for the free drug and the AgDC.

\section{In vivo screening of conjugates}

In vivo efficacy of PLP-DEX in the treatment of EAE was determined through subcutaneous administration of the conjugate as well as component treatments at a dose equivalent to 200 nmol DEX. Treatments were administered in $40 \mathrm{mg} / \mathrm{mL}$ mannitol on days 4,7 , and 10 with $\mathrm{n}=9$ prior to day 14 and $\mathrm{n}=4$ after day 14 . Five mice in each treatment group were chosen at random and euthanized at peak of disease in order to assess splenocyte response to $25 \mu \mathrm{M}$ PLP rechallenge. This in vivo schedule allows for determination of the effectiveness of $\mathrm{AgDCs}$ in ameliorating EAE clinical symptoms as well as mechanistic insight into changes in cellular expression after treatment.

In vivo clinical score data indicates the importance of conjugation of DEX to the antigen of interest (Figure 7). EAE mice treated with the AgDC demonstrated no clinical symptoms throughout the 25 day study (Figure 7B). This represents a vast improvement over the free drug, DEX (Figure 7A), which appears to have no clinical effect in the EAE model at a 200 nmol dose. Similar to the AgDC, administration of a combination of the components, PLP and DEX, ameliorates symptoms and appears to delay the onset of paralysis in EAE mice (Figure 7B); however, this co-administration is less effective than co-delivery through direct conjugation. Additionally, co-administration of PLP and DEX as a mixture resulted in delayed symptom onset in those mice which developed disease (Table 1). Treatment with individual components, PLP or DEX, had no significant effect in reducing EAE symptoms (Figure 7A). Similar trends are observed in animal weight data (Figure 9), in which weight gain is associated with a healthy mouse and weight loss correlates to symptom severity. In combination therapies (Figure 9B), continuous weight gain is observed throughout the duration of the study. Conversely, mice treated with individual components as well as mannitol (Figure 9A) displayed significant weight loss leading up to disease remission.

As previously stated, 5 mice were euthanized on day 14 in order to assess cellular differences due to the various in vivo treatments (Figures 10 and S2-S8). Of note, at day 14 a small but significant reduction in GM-CSF (Figure 10A), an inflammatory cytokine, was observed for combination therapies. Additionally, detection of IL-2 (Figure 10B), a cytokine associated with T-cell proliferation, was greatly diminished in combination therapies as well as the PLP control. These results demonstrate the early immunosuppressive effects of combination treatments containing DEX. Comparisons of GM-CSF and IL-2 between the mixture of PLP and DEX and the AgDC revealed no significant differences between coadministration and co-delivery at this stage of treatment. One notable difference between these treatment groups in cytokine expression was observed in IL-10 (Figure 10C), in which the mixture treatment group experienced a significant reduction in IL-10 expression compared to control. This result was not observed in the conjugate treatment group and may account for the differences in efficacy observed in vivo by maintaining an anti-inflammatory effector cell population. Numerous other cytokines were analyzed; however, no significant 
changes associated with treatment efficacy were observed in the expression of these cytokines (Figures S2-S8).

\section{Discussion}

Clinical researchers attempting to treat autoimmune diseases such as MS have historically focused on either administering drugs to suppress and/or modulate the immune response (e.g. classic immunosuppression) or, more recently, ASIT (e.g. long treatment regimens of low doses of antigen or altered antigen analogs). ${ }^{16}$ Currently, potent immunomodulatory drugs represent the core treatment options for MS patients, but these drugs act in a nonspecific manner and may eliminate or suppress healthy immune cell populations. Conversely, although ASIT is relatively safe, this approach has not yielded sufficient efficacy to suppress autoimmunity. ${ }^{7-9}$ Through direct conjugation of potent immunomodulators to self-antigens, targeted immunosuppression of self-reactive populations may boost the efficacy of ASIT and diminish the side effects associated with immunosuppressants.

The encephalitogenic peptide selected as the targeting moiety is a portion of the intracellular loop ${ }^{17}$ of the full-length transmembrane protein, PLP139-151. Previous studies have shown the impact of PLP139-151 sequence variability on TCR binding affinity ${ }^{18}$, highlighting the influence of side chain interactions in the major histocompatibility class 2 binding pocket. Therefore, disrupting interactions through traditional synthetic methods targeting side chain residues of the native sequence were hypothesized to alter conjugate binding and/or uptake. To circumvent these potential deleterious effects, all modifications to the targeting antigen occurred through the $N$-terminal amino acid and were installed via heterobifunctional linkers as the final step of solid-phase peptide synthesis, prior to cleavage from the resin. Linker length, flexibility, and stability were key AgDC design considerations. The overarching strategy utilizing copper-catalyzed azide alkyne cycloaddition (CuAAC) enabled rapid and efficient synthesis of PLP-DEX (Figure 2) and related conjugates.

Data reported here support a growing base of literature highlighting the benefits of delivering auto-antigen alongside immunomodulators. Although historically categorized as an immunosuppressant, DEX is now known to skew cellular responses towards immune tolerance via multiple mechanisms. ${ }^{19-22}$ For example, DEX was shown to enhance CTLA-4 expression during $\mathrm{T}$ cell activation, thus countering the typical $\mathrm{B} 7 / \mathrm{CD} 28$ pro-inflammatory costimulatory signal. ${ }^{22}$ DEX also inhibited IL-12 secretion by dendritic cells and increased FoxP3 expression in naïve T lymphocyte co-cultures. ${ }^{20} \mathrm{DEX}$ was reported to induce expression of indoleamine 2-3 dioxygenase (IDO), an enzyme implicated in T cell tolerance ${ }^{21}$ has been used to create 'tolerogenic dendritic cells' (defined as high TLR-2, CXCR4, and CCR7 expression levels) from healthy donors. ${ }^{19}$

Because of these pluripotent mechanisms, some have tested DEX as an adjuvant that can be co-administered along with antigen as a means to induce antigen-specific immune tolerance. 20, 23-24 Previously, Kang and colleagues demonstrated desensitization specific to OVA323339 peptide after co-administration of this peptide alongside DEX. ${ }^{25}$ Additionally, applying this strategy of co-administration to the NOD autoimmune diabetes model protected nearly 
all mice from disease induction throughout the study period, suggesting these therapies may be adapted for application to numerous autoimmune diseases with known auto-antigens. ${ }^{25}$ Further development of this treatment strategy by Peine et al. utilized acetalated dextran particles as a co-delivery vehicle for a myelin oligodendrocyte glycoprotein (MOG) peptide and DEX in EAE, and administration of these microparticles to EAE mice after disease onset resulted in significant reduction in disease severity when both components were present. ${ }^{26}$ With such striking differences in vivo between combination therapies and free DEX, our findings highlight the advantages associated with delivery of an immunosuppressive drug in the context of the auto-antigens of interest. Furthermore, conjugation of DEX to PLP139-151 may maximize co-delivery of auto-antigen and immunomodulator in larger organisms, enhancing drug potency as demonstrated and potentially limiting off-target effects.

\section{Conclusions}

Antigen-drug conjugates (AgDCs) represent a novel class of therapeutics with broad applicability to a variety of antigen-specific autoimmune disorders. By combining the benefits of two traditional treatment approaches, ASIT and immunomodulatory therapy, AgDCs employ a synergistic approach by conjugating the antigen to the immunomodulatory agent. The enhanced specificity associated with therapeutics of this class may help to minimize global immunosuppression often observed with immunomodulatory small molecule treatments. This publication serves as an introduction to the modular synthetic design achieved in our lab and demonstrates both efficacy and safety of in vivo AgDC treatment in a mouse model of MS with which the autoreactive antigen is identified. The EAE model provides a valuable system for the initial testing of AgDCs as induction and treatment of the disease can be achieved utilizing a single antigen epitope, in this case PLP139-151. Subcutaneous treatment with free DEX at a $200 \mathrm{nmol}$ dose appears to have little effect compared to control on modifying the disease course. EAE mice treated in vivo with PLP-DEX; however, demonstrated no onset of disease over a 25 day period and appeared healthy throughout the study, which demonstrates the efficacy achievable with codelivery of the antigen and immunomodulator. The modular fashion of this AgDC approach, coupled with the consistency and specificity of the chemistries employed, make AgDCs a potential disease-specific therapeutic class for autoimmune disorders.

\section{Supplementary Material}

Refer to Web version on PubMed Central for supplementary material.

\section{Acknowledgements}

We gratefully acknowledge support from the National Institutes of Health Graduate Training Program in Dynamic Aspects of Chemical Biology Grant (T32 GM008545) from the National Institutes of General Medical Sciences (C.J.P. and M.A.L.), and the Howard Rytting pre-doctoral fellowship from the Department of Pharmaceutical Chemistry at the University of Kansas (C.J.P.). Additionally, we would like to acknowledge support from the National Institutes of Health Biotechnology Training Grant (NIH0073415) (M.A.C.). We would also like to thank the KU NMR facility and the KU Macromolecule and Vaccine Stabilization Center (MVSC) for collaboration and instrument use. As well as, support for the NMR instrumentation was provided by NIH Shared Instrumentation Grant \# S10RR024664 and NSF Major Research Instrumentation Award \# 1625923. We also thank Tom Prisinzano for the useful discussions on synthetic strategy. 


\section{References}

1. Murphy KM, Janeway's Immunobiology. 8th ed.; Garland Science: 2012.

2. Perl A, Autoimmunity Methods and Protocols. 2nd ed.; Humana Press: 2012.

3. Compston AC,A, Multiple Sclerosis. Lancet 2002, 359, 1221-1231. [PubMed: 11955556]

4. Dendrou CA; Fugger L; Friese MA, Immunopathology of multiple sclerosis. Nat Rev Immunol 2015, 15 (9), 545-58. [PubMed: 26250739]

5. Podbielska M; Banik NL; Kurowska E; Hogan EL, Myelin recovery in multiple sclerosis: the challenge of remyelination. Brain Sci 2013, 3 (3), 1282-324. [PubMed: 24961530]

6. Karussis D, Immunotherapy of multiple sclerosis: the state of the art. BioDrugs 2013, 27 (2), 11348. [PubMed: 23423844]

7. Bielekova B; Goodwin B; Richert N; Cortese I; Kondo T; Afshar G; Gran B; Eaton J; Antel J; Frank JA; McFarland HF; Martin R, Encephalitogenic potential of the myelin basic protein peptide (amino acids 83-99) in multiple sclerosis: Results of a phase II clinical trial with an altered peptide ligand. Nature medicine 2000, 6, 1167.

8. Kappos L; Comi G; Panitch H; Oger J; Antel J; Conlon P; Steinman L; Comi G; Kappos L; Oger J; Panitch H; Rae-Grant A; Castaldo J; Eckert N; Guarnaccia JB; Mills P; Johnson G; Calabresi PA; Pozzilli C; Bastianello S; Giugni E; Witjas T; Cozzone P; Pelletier J; Pöhlau D; Przuntek H; Hoffmann V; Bever C Jr; Katz E; Clanet M; Berry I; Brassat D; Brunet I; Edan G; Duquette P; Radue E-W; Schött D; Lienert C; Taksaoui A; Rodegher M; Filippi M; Evans A; Bourgouin P; Zijdenbos A; Salem S; Ling N; Alleva D; Johnson E; Gaur A; Crowe P; Liu X-J, Induction of a non-encephalitogenic type $2 \mathrm{~T}$ helper-cell autoimmune response in multiple sclerosis after administration of an altered peptide ligand in a placebo-controlled, randomized phase II trial. Nature medicine 2000, 6, 1176.

9. Freedman MS; Bar-Or A; Oger J; Traboulsee A; Patry D; Young C; Olsson T; Li D; Hartung H-P; Krantz M; Ferenczi L; Verco T, A phase III study evaluating the efficacy and safety of MBP8298 in secondary progressive MS. Neurology 2011, 77 (16), 1551-1560. [PubMed: 21975206]

10. Miller SD; Karpus WJ, Experimental autoimmune encephalomyelitis in the mouse. Curr Protoc Immunol 2007, Chapter 15, Unit 151.

11. Miyagaki T; Fujimoto M; Sato S, Regulatory B cells in human inflammatory and autoimmune diseases: from mouse models to clinical research. International Immunology 2015, 27 (10), 495504. [PubMed: 25957264]

12. Northrup L; Sullivan BP; Hartwell BL; Garza A; Berkland C, Screening Immunomodulators To Skew the Antigen-Specific Autoimmune Response. Molecular Pharmaceutics 2017, 14 (1), 66-80. [PubMed: 28043135]

13. Brandl C; Haas C; d'Argouges S; Fisch T; Kufer P; Brischwein K; Prang N; Bargou R; Suzich J; Baeuerle PA; Hofmeister R, The effect of dexamethasone on polyclonal T cell activation and redirected target cell lysis as induced by a CD19/CD3-bispecific single-chain antibody construct. Cancer immunology, immunotherapy : CII 2007, 56 (10), 1551-63. [PubMed: 17310380]

14. Palma L; Sfara C; Antonelli A; Magnani M, Dexamethasone restrains ongoing expression of interleukin-23p19 in peripheral blood-derived human macrophages. BMC pharmacology 2011, 11, 8. [PubMed: 21791059]

15. Peakman M; Dayan CM, Antigen-specific immunotherapy for autoimmune disease: fighting fire with fire? Immunology 2001, 104 (4), 361-6. [PubMed: 11899420]

16. Cox L; Compalati E; Kundig T; Larche M, New directions in immunotherapy. Current allergy and asthma reports 2013, 13 (2), 178-95. [PubMed: 23315329]

17. Greer JM, Autoimmune T-cell reactivity to myelin proteolipids and glycolipids in multiple sclerosis. Mult Scler Int 2013, 2013, 151427. [PubMed: 24312732]

18. V K Kuchroo JMG, D Kaul G Ishioka, A Franco, A Sette, R A Sobel, M B Lees, A single TCR antagonist peptide inhibits experimental allergic encephalomyelitis mediated by a diverse $\mathrm{T}$ cell repertoire. J. Immunol 1994, 153, 3326-3336. [PubMed: 7522258]

19. Garcia-Gonzalez P; Morales R; Hoyos L; Maggi J; Campos J; Pesce B; Garate D; Larrondo M; Gonzalez R; Soto L; Ramos V; Tobar P; Molina MC; Pino-Lagos K; Catalan D; Aguillon JC, A short protocol using dexamethasone and monophosphoryl lipid A generates tolerogenic dendritic 
cells that display a potent migratory capacity to lymphoid chemokines. Journal of translational medicine 2013, 11, 128. [PubMed: 23706017]

20. Gong YB; Huang YF; Li Y; Han GC; Li YR; Wang DJ; Du GP; Yu JF; Song J, Experimental study of the mechanism of tolerance induction in dexamethasone-treated dendritic cells. Medical science monitor : international medical journal of experimental and clinical research 2011, 17 (5), BR12531. [PubMed: 21525800]

21. Grohmann U; Volpi C; Fallarino F; Bozza S; Bianchi R; Vacca C; Orabona C; Belladonna ML; Ayroldi E; Nocentini G; Boon L; Bistoni F; Fioretti MC; Romani L; Riccardi C; Puccetti P, Reverse signaling through GITR ligand enables dexamethasone to activate IDO in allergy. Nature medicine 2007, 13 (5), 579-86.

22. Xia M; Gasser J; Feige U, Dexamethasone enhances CTLA-4 expression during T cell activation. Cellular and molecular life sciences : CMLS 1999, 55 (12), 1649-56. [PubMed: 10526580]

23. Zheng G; Zhong S; Geng Y; Munirathinam G; Cha I; Reardon C; Getz GS; van Rooijen N; Kang Y; Wang B; Chen A, Dexamethasone promotes tolerance in vivo by enriching CD11clo CD401o tolerogenic macrophages. European journal of immunology 2013, 43 (1), 219-27. [PubMed: 23001956]

24. Unger WW; Laban S; Kleijwegt FS; van der Slik AR; Roep BO, Induction of Treg by monocytederived DC modulated by vitamin D3 or dexamethasone: differential role for PD-L1. European journal of immunology 2009, 39 (11), 3147-59. [PubMed: 19688742]

25. Kang Y; Xu L; Wang B; Chen A; Zheng G, Cutting Edge: Immunosuppressant as Adjuvant for Tolerogenic Immunization. The Journal of Immunology 2008, 180 (8), 5172-5176. [PubMed: 18390698]

26. Peine KJ; Guerau-de-Arellano M; Lee P; Kanthamneni N; Severin M; Probst GD; Peng H; Yang Y; Vangundy Z; Papenfuss TL; Lovett-Racke AE; Bachelder EM; Ainslie KM, Treatment of Experimental Autoimmune Encephalomyelitis by Codelivery of Disease Associated Peptide and Dexamethasone in Acetalated Dextran Microparticles. Molecular Pharmaceutics 2014, 11 (3), 828-835. [PubMed: 24433027] 

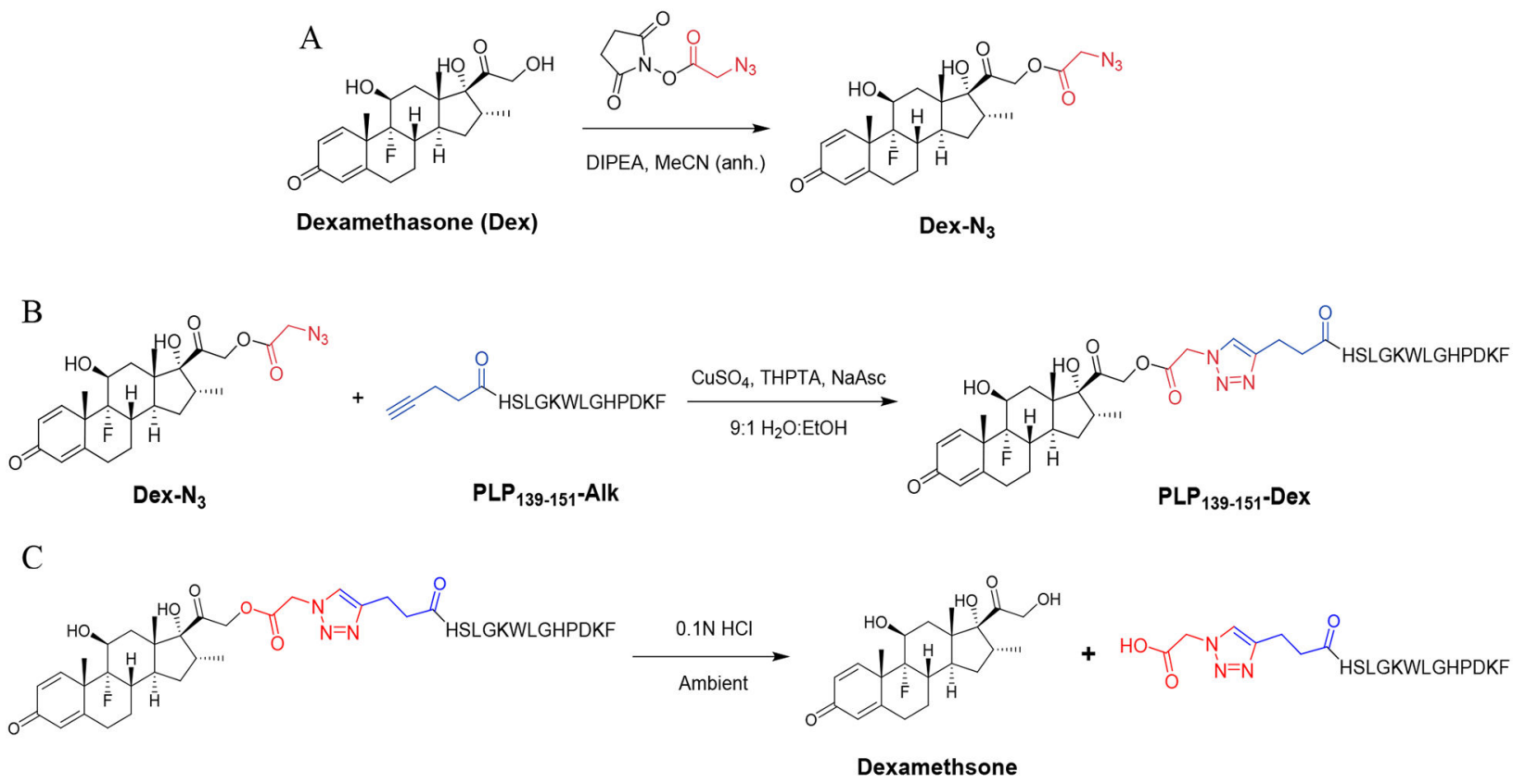

Figure 1:

(A) Reaction scheme for the synthesis of DEX-N $\mathrm{N}_{3}$. (B) Reaction scheme for the synthesis of PLP-DEX. (C) Degradation of PLP-DEX to release parent drug, DEX. 
0.85

0.80

0.75

0.70

0.65

0.60

0.55

0.50

₹ 0.45

0.35

0.30

0.25

0.20

0.15

0.10

0.05

0.00

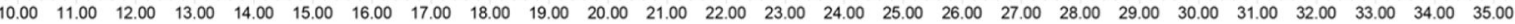
DEX-N

\section{PLP-Alk}

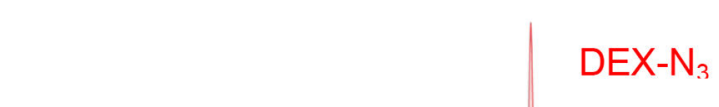

\section{PLP-DEX}

Figure 2:

Representative analytical HPLC chromatogram showing the starting materials, PLP-Alk, and DEX-N ${ }_{3}$, along with the purified reaction product, PLP-DEX. 


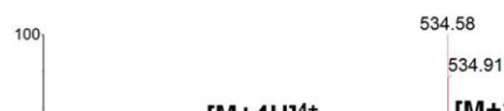

534.91

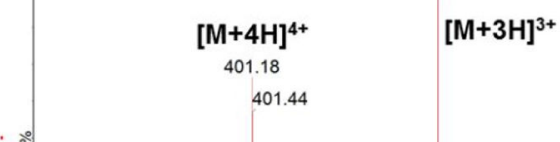

PLP-Alk

Exact Mass: $1601.82 \mathrm{Da}$
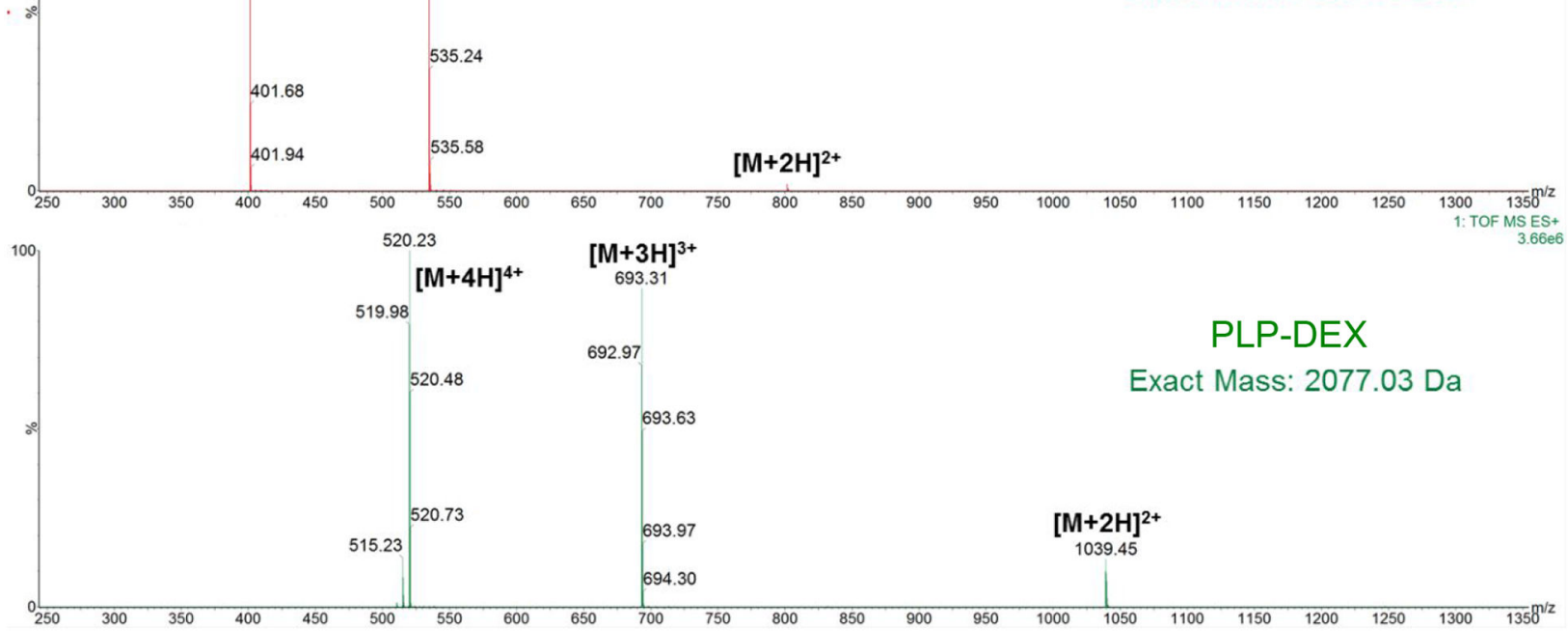

Figure 3:

Representative mass spectral characterization showing multiple charge states of the starting peptide and the antigen-drug conjugate, PLP-DEX. 

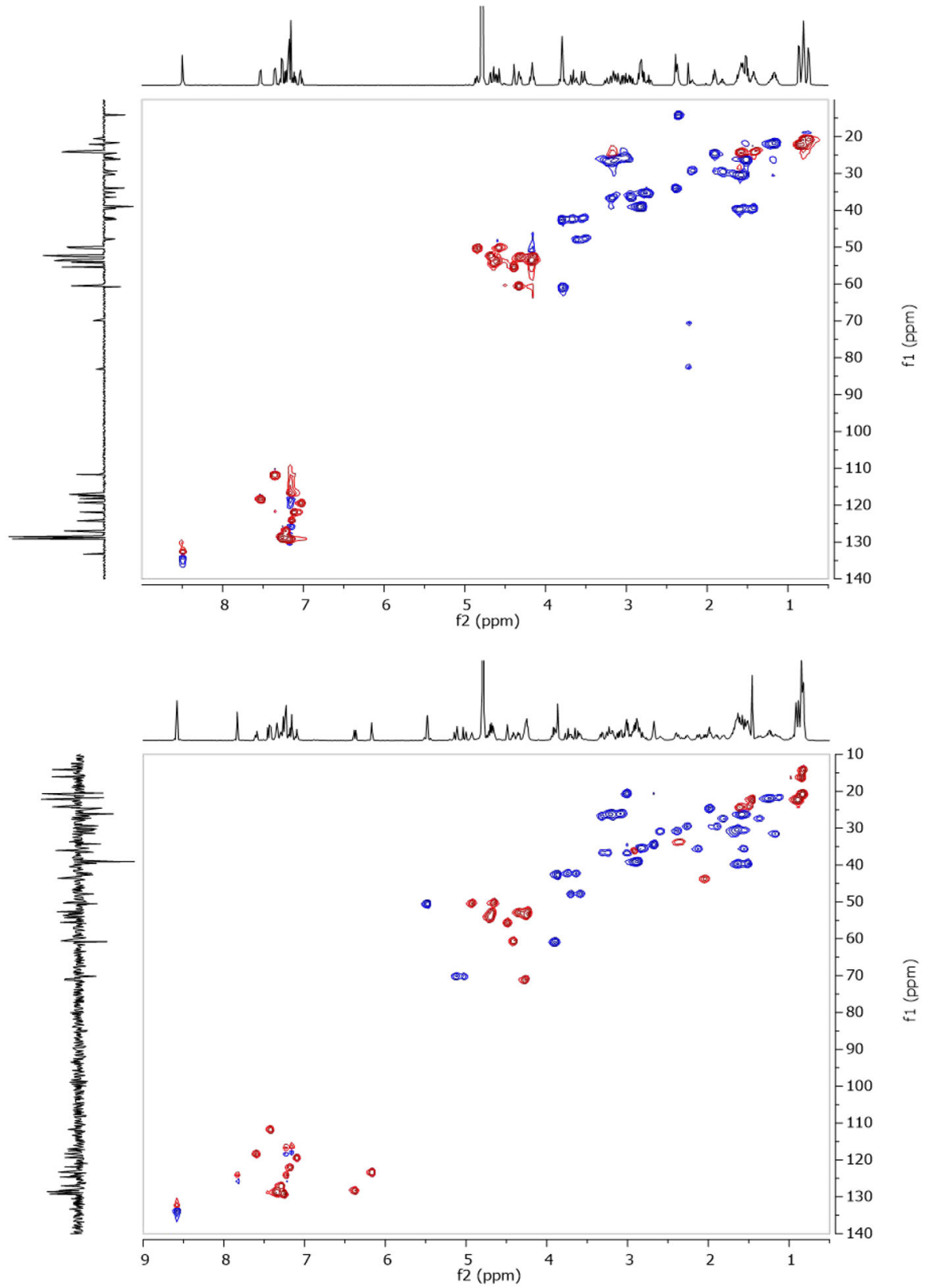

Figure 4:

${ }^{1} \mathrm{H} /{ }^{13} \mathrm{C}$ HSQC NMR data for PLP139-151-Alk (top) and PLP139-151-DEX (bottom). 


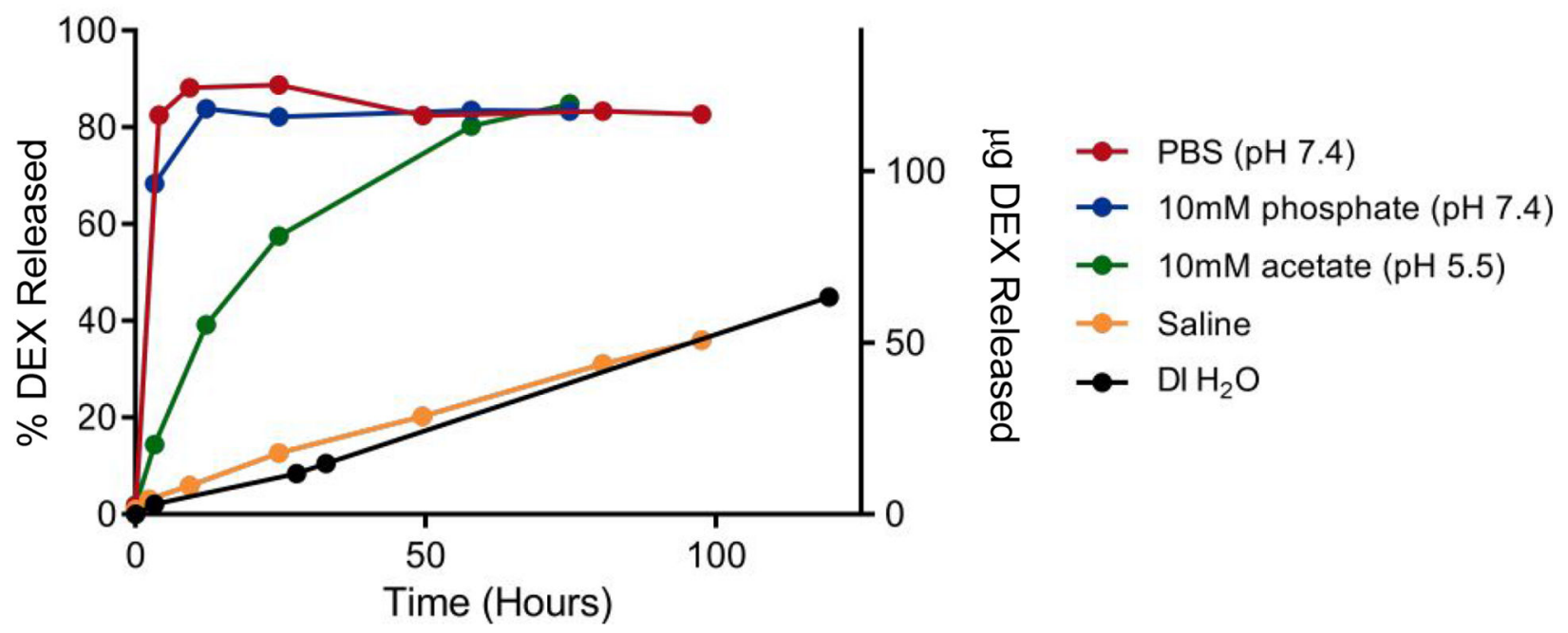

Figure 5:

Release of DEX from PLP139-151-DEX as a function of time and buffer, at $37^{\circ} \mathrm{C}$. Starting peptide concentration was $1 \mathrm{mg} / \mathrm{mL}$, corrected for potency, and quantified via linear calibration curve. 


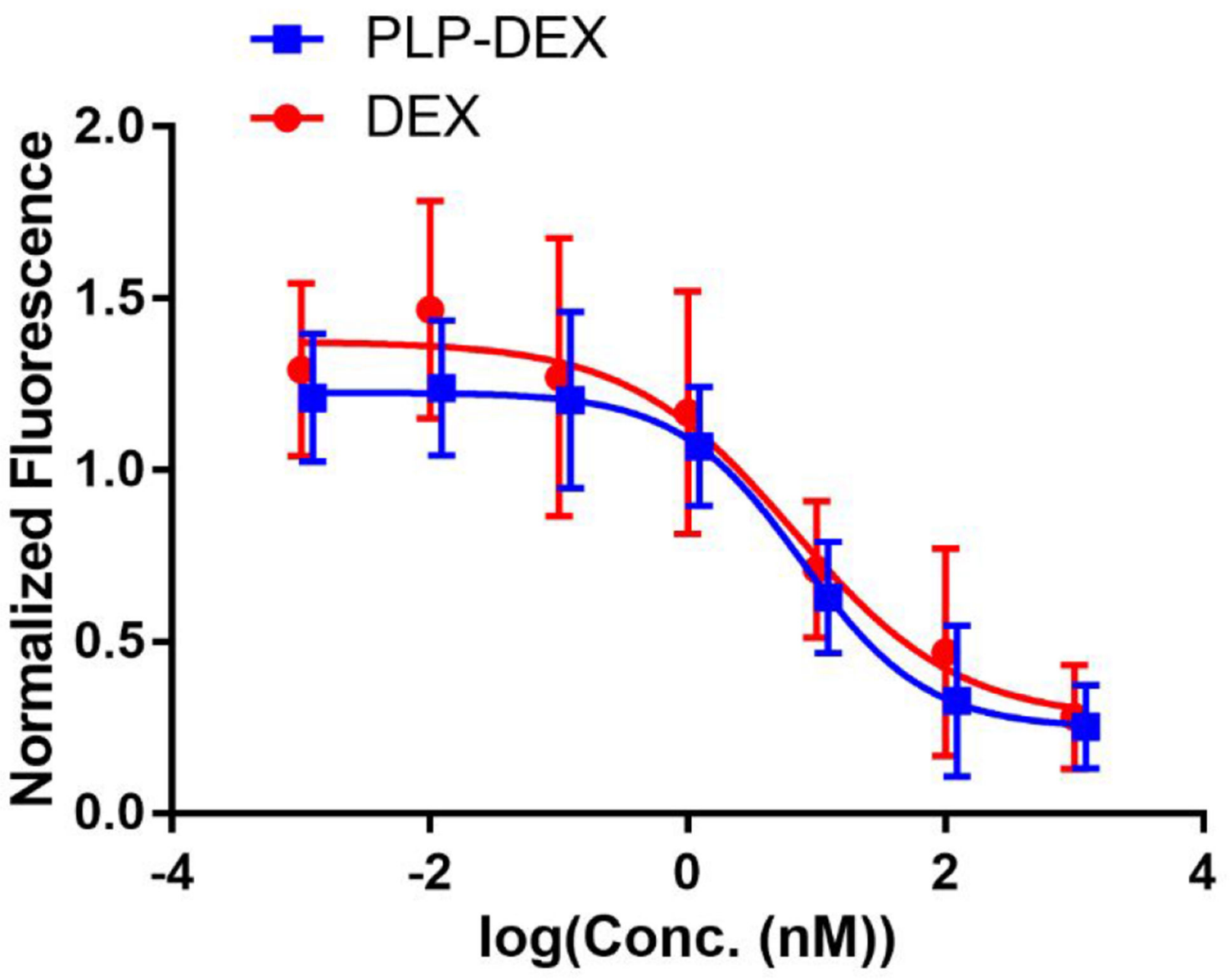

Figure 6:

Resorufin fluorescence of EAE splenocytes treated with free DEX or PLP-DEX for 120 hours $(n=6)$. These data are normalized to untreated EAE splenocytes. Calculated IC50s for these treatments are $6.84+/-4.18 \mathrm{nM}$ and $7.55+/-2.80 \mathrm{nM}$ for DEX and PLP-DEX, respectively. Reported values are with standard error. 

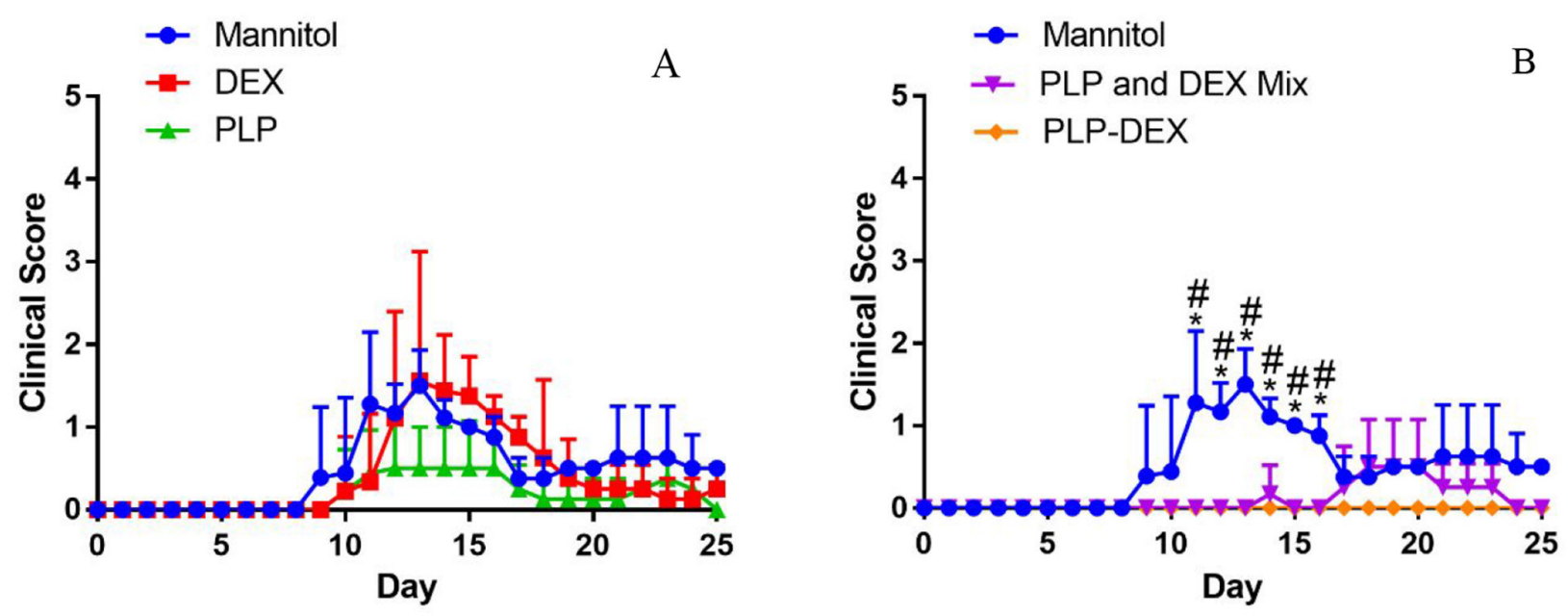

Figure 7:

Clinical score data for EAE mice treated in vivo with (A) free DEX and free PLP139-151 as well as (B) a mixture of free DEX and PLP139-151 and the PLP-DEX conjugate.

Treatments were administered subcutaneously on days 4, 7, and 10 at doses of $200 \mathrm{nmol}$ DEX basis. $\mathrm{N}=9$ until day 14 and $\mathrm{n}=4$ after day 14 . Five mice were chosen at random from each group on day 14 for euthanasia in order to assess splenocyte differences in treatment groups around peak of disease. Data provided is mean \pm SD. $* p<0.05$ for Mannitol vs PLPDEX and \# p $<0.05$ for Mannitol vs PLP and DEX Mix. 


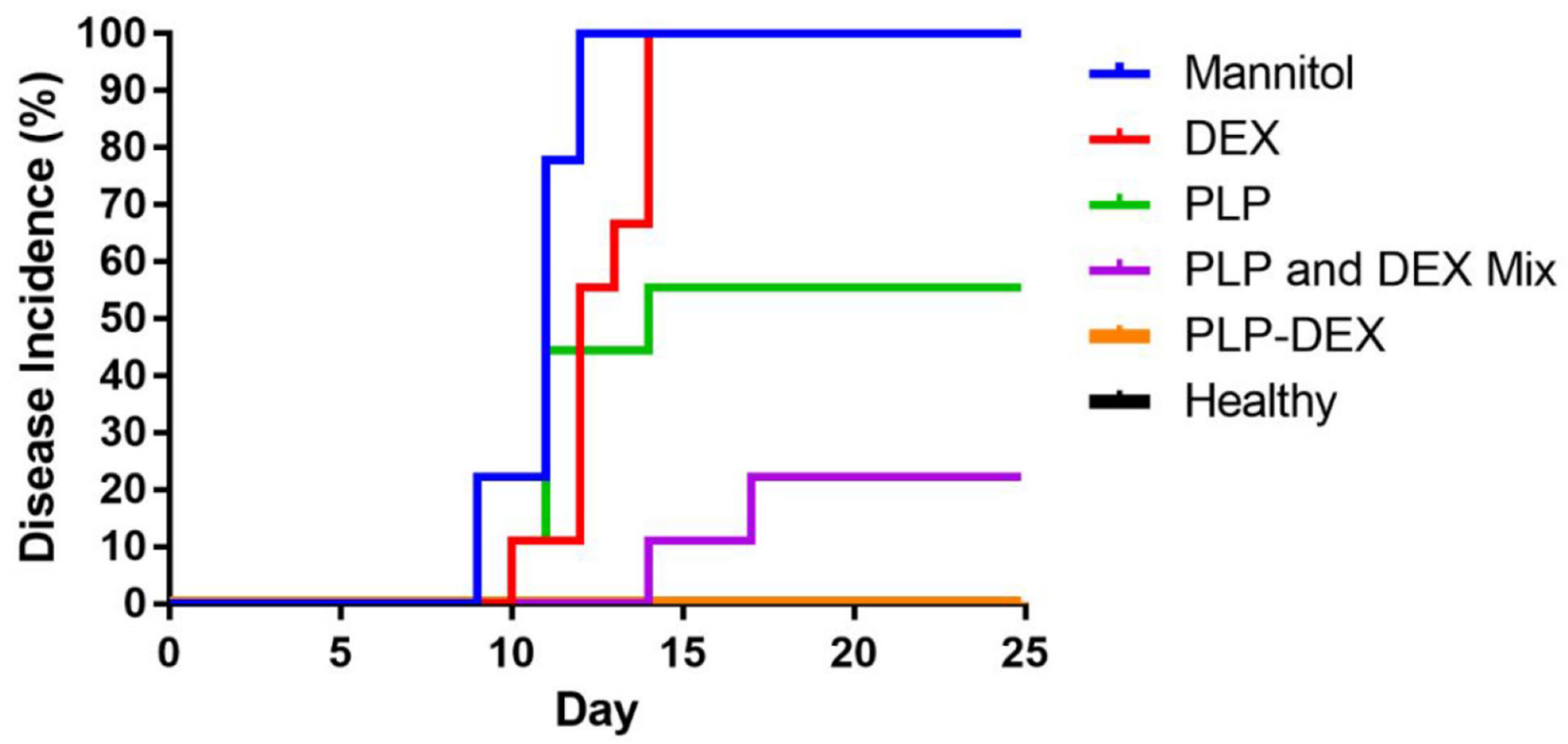

Figure 8:

Percent disease incidence for all treatment groups where disease onset is characterized by a c.s. $\geq 1$. $N=9$ until day 14 and $n=4$ after day 14 . Five mice were chosen at random from each group on day 14 for euthanasia in order to assess splenocyte differences in treatment groups around peak of disease. 

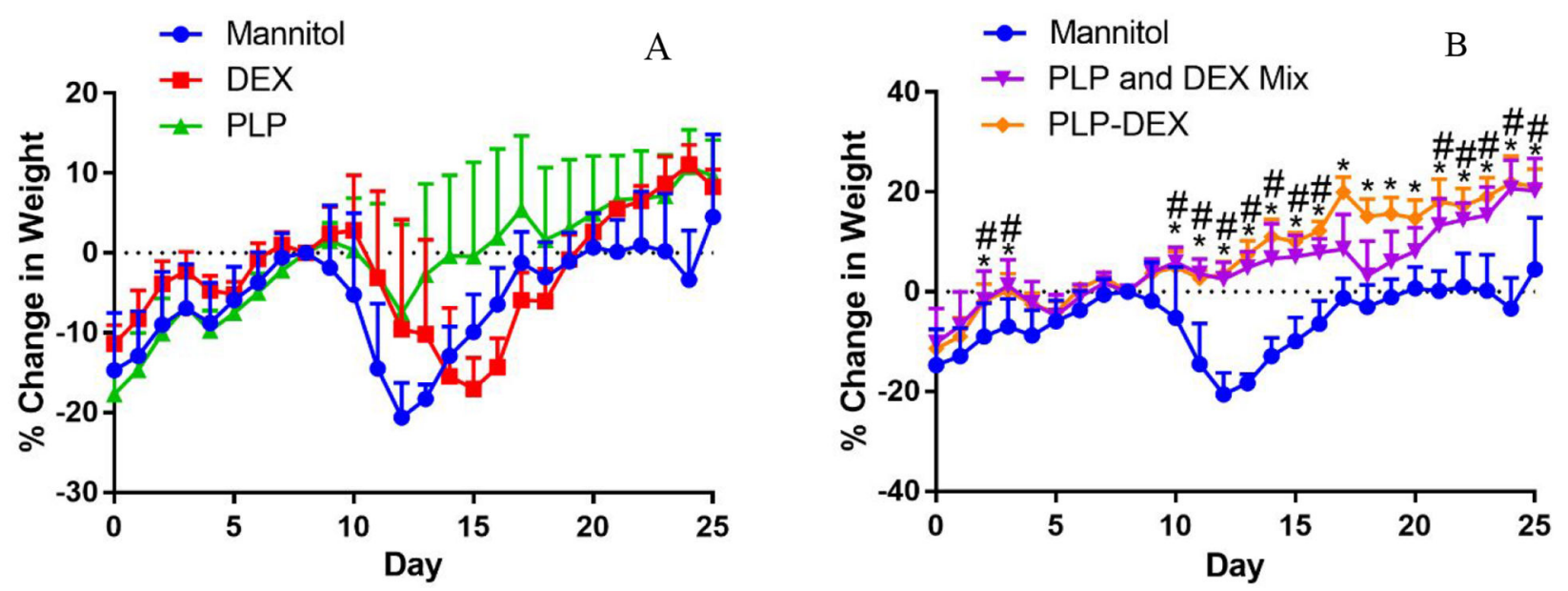

Figure 9:

Mouse weight data for EAE mice treated in vivo with (A) free DEX and free PLP139-151 as well as (B) a mixture of free DEX and PLP139-151 and the PLP-DEX conjugate. Data are normalized to the individual mouse weight at day 8 (symptom onset). Treatments were administered subcutaneously on days 4,7 , and 10 at doses of $200 \mathrm{nmol}$ DEX basis. $\mathrm{N}=9$ until day 14 and $n=4$ after day 14 . Five mice were chosen at random from each group on day 14 for euthanasia in order to assess splenocyte differences in treatment groups around peak of disease. ${ }^{*} \mathrm{p}<0.05$ for Mannitol vs PLP-DEX and \# $\mathrm{p}<0.05$ for Mannitol vs PLP and DEX Mix. 

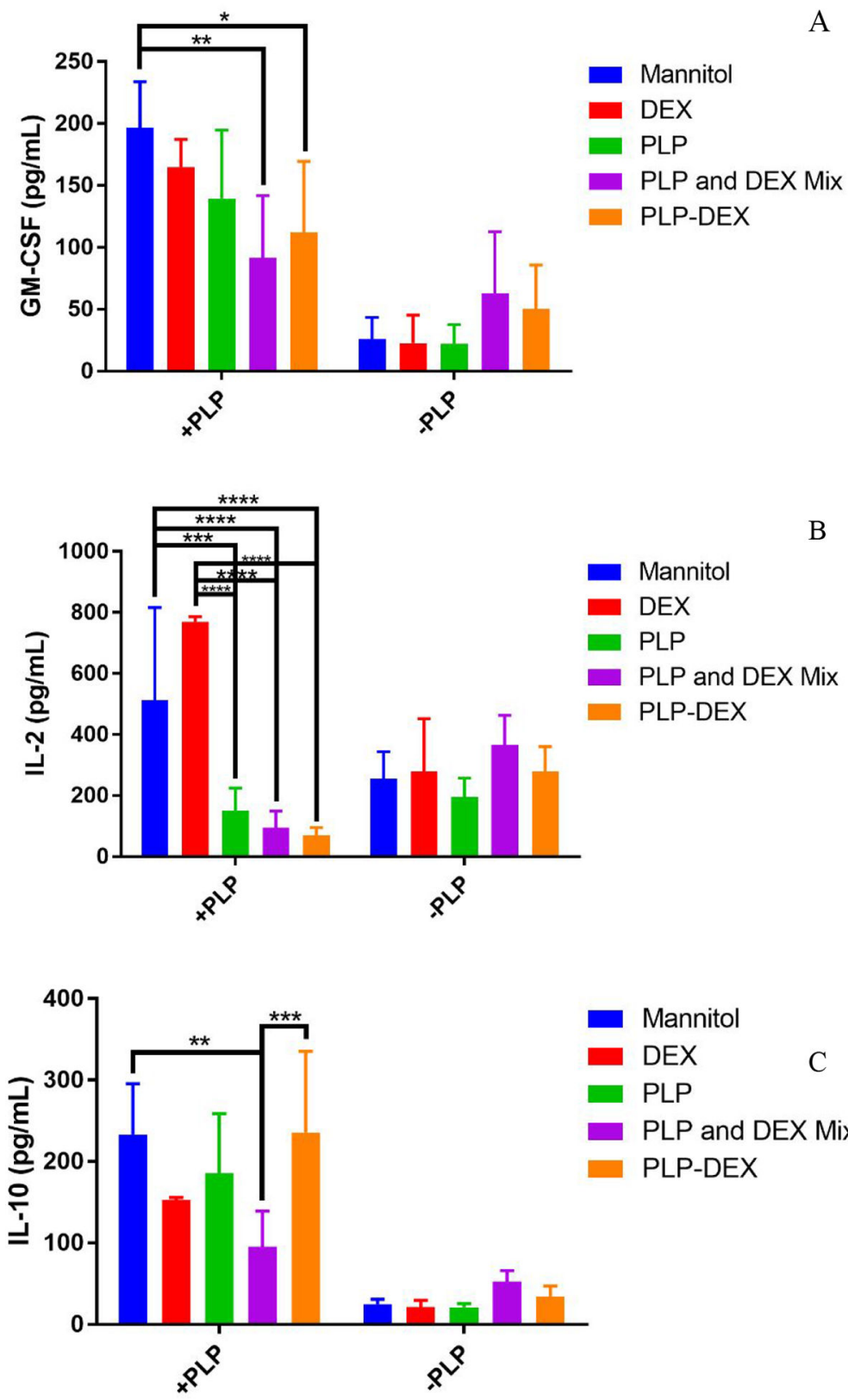

Figure 10:

Levels of (A) GM-CSF (B) IL-2 and (C) IL-10 were observed at peak of disease (day 14) after isolated splenocytes from in vivo treated mice were incubated for 96 hours with $25 \mu \mathrm{M}$ PLP. $\mathrm{N}=5, * \mathrm{p}<0.05, * * \mathrm{p}<0.01, * * * \mathrm{p}<0.001, * * * * \mathrm{p}<0.0001$ 
Table 1:

Disease incidence rate and mean day of disease onset \pm SD for each treatment group. Incidence rate is represented as a fraction of total mice per treatment group with clinical score $\geq 1$. Mean day of onset is calculated only for symptomatic animals.

\begin{tabular}{|l|c|c|c|c|c|}
\hline & Mannitol & DEX & PLP & PLP and DEX mix & PLP-Dex \\
\hline IncidenceRate(Fraction) & $9 / 9$ & $9 / 9$ & $5 / 9$ & $2 / 9$ & $0 / 9$ \\
\hline Mean Day of Onset \pm SD & $10.8 \pm 1.1$ & $12.6 \pm 1.3$ & $11.4 \pm 1.5$ & $15.5 \pm 2.1$ & - \\
\hline
\end{tabular}

